\title{
Individual particle handling in a microfluidic system based on parallel laser trapping
}

\author{
Philippe Hamel, ${ }^{1, *}$ Bastien Rachet, ${ }^{1}$ Michael Werner, ${ }^{2}$ Mathieu Grossenbacher, ${ }^{1}$ \\ Horst Vogel, ${ }^{2}$ Martin Forrer, ${ }^{3}$ Peter Ryser, ${ }^{4}$ and René P. Salathé ${ }^{1}$ \\ ${ }^{1}$ Applied Optics Laboratory, School of Engineering, EPFL, Station 17, CH-1015 Lausanne, Switzerland \\ ${ }^{2}$ Laboratory of Physical Chemistry of Polymers and Membranes, School of Engineering, EPFL, \\ Station 17, CH-1015 Lausanne, Switzerland \\ ${ }^{3}$ FISBA OPTIK AG, Rorschacherstrasse 268, CH-9016 St. Gallen, Switzerland \\ ${ }^{4}$ Tecan AG, Seestrasse 103, CH-8708 Männedorf, Switzerland \\ *Corresponding author: philippe.hamel@epfl.ch
}

Received April 18, 2011; revised July 12, 2011; accepted July 18, 2011; posted July 18, 2011 (Doc. ID 146031); published August 11, 2011

\begin{abstract}
We present an optical trapping system combining individually addressable multiple laser traps with fluorescence spectroscopy. An in-line set of 64 near-IR laser diodes is used to create a line of individually addressable traps inside a microfluidic chip. This system is completed by an excitation/detection line for spectrally resolved fluorescence imaging of trapped particles. Highly parallel trapping in a constant flow (up to a few millimeters per second), fast particle handling rates (up to a few particles per second), and the possibility of recording fluorescence spectra of trapped objects lead to a performing bioanalytical platform, e.g., for highly parallel analysis and sorting. (c) 2011 Optical Society of America

OCIS codes: $230.0230,170.3890,300.6280,350.4855$.
\end{abstract}

Since their first description [1,2], optical trapping techniques have evolved considerably to become powerful and versatile tools for various applications [3-5]. Among these, several techniques [ $\underline{6}-9]$ perform multiple trapping and/or micromanipulation of particles within enclosed liquid chambers. Multiple traps are usually generated from a single laser beam using time or space multiplexing techniques, based on, e.g., fast scanners $[\underline{10}, 11]$ or spatial light modulators [12]. Here, the laser power available per trap decreases with an increasing number of traps. Multiplexing also imposes time constraints with respect to the on/ off switching of individual traps. These limitations can be avoided by using multiple laser sources. Vertical cavity surface emitting laser (VCSEL) arrays have been proposed by Ogura et al. [13]. But the limited VCSEL output power did not allow demonstration of three-dimensional (3D) trapping. Boer et al. demonstrated simultaneous trapping in microfluidics using four diode lasers [14]. The number of traps could in principle be increased in such configurations, but extension to a large number of traps would result in cumbersome devices.

Here, we present a system based on a diode laser array that allows the simultaneous generation of 64 optical traps inside a microfluidic system. Each diode of the array can be addressed individually, allowing individual adjustment of the strength of each trap. Micrometer-sized objects in the flow can be trapped or released in a controlled manner, offering the possibility of sorting them within the microfluidic device. The linear arrangement of the traps also offers the possibility of spectrally resolving the fluorescence of individual trapped particles over time. In combination with the spatiotemporal control provided by microfluidics, our platform has great potential for single live cell analysis.

The experimental arrangement is shown schematically in Fig. 1. Multiple optical trapping is achieved via a laser diode array designed and provided for industrial printing applications (FISBA OPTIK). The 64 stripe geometry la- sers with a distance of $170 \mu \mathrm{m}$ between diodes can be driven separately with a dedicated electronic circuit. This allows individual on/off switching and adaptation of the strength of the 64 lasers. The peak emission wavelength of the diodes is at $830 \mathrm{~nm}$, and the maximum emission power of each diode is $100 \mathrm{~mW}$, with a variation of less than $5 \%$ over the whole array. The emission of the array is collimated with a propriety cylindrical optics, a dichroic beam splitter BS1, and a relay lens of $200 \mathrm{~mm}$ focal length L1 into a 1.25 NA microscope objective (MO) of an inverse microscope (Leica DM IL). Trapping occurs in the focal plane of the $\mathrm{MO}$, which is placed into the $500 \mu \mathrm{m}$ wide, $50 \mu \mathrm{m}$ deep main channel of a polymer microfluidic system that is described later in this Letter.

The system also features fluorescence excitation and detection with linear geometry in order to perform simultaneous measurements on trapped particles. As a proof of principles, $6 \mu \mathrm{m}$ diameter polystyrene beads coated with a red fluorescent marker have been used in these experiments. Only every second laser diode was excited

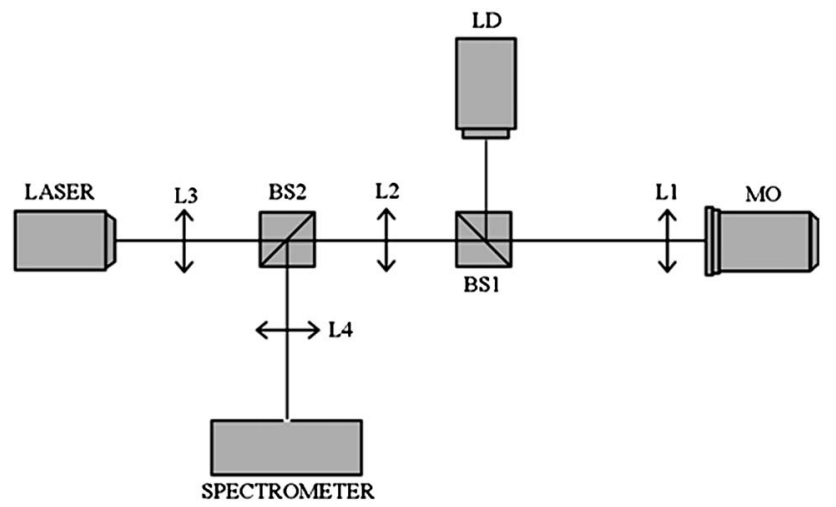

Fig. 1. Simplified schematic description of the system. LD, laser diodes; L1-L4, lenses; BS1, BS2, beam splitters; MO, microscope objective. Trapping and fluorescence excitation occur at the focal plane of the MO. 
during this experiment, leading to a distance between the traps of approximately $10 \mu \mathrm{m}$. The fluorescence of the trapped particles is excited with a He-Ne laser operated at a wavelength of $633 \mathrm{~nm}$. The shape of the beam is turned into a line via a cylindrical lens L3 $(100 \mathrm{~mm}$ focal length) and then propagated to the MO through the two dichroic beam splitters BS1 and BS2 and relay lenses L1 and L2 (100 mm focal length). The elliptic focal shape of the He-Ne beam is adjusted in a way that the major axis coincides with the line of foci from the diode laser array. The fluorescence light emitted from the trapped particles is collimated by the MO, the two beamsplitters BS1 and BS2, and the two relay lenses L1 and L2. It is then focused by an additional lens $\mathrm{L} 4$ of $100 \mathrm{~mm}$ focal length onto a $50 \mu \mathrm{m}$ wide entrance slit of a spectrometer. The size of the diode array's image on the spectrometer $(6.4 \mathrm{~mm})$ is smaller than the size of the cooled CCD $576 \times 384$ pixel array (EEV) at the spectrometer's exit plane $(8.5 \mathrm{~mm})$, i.e., the fluorescence emission from all 64 trapped particles can be recorded in parallel by proper adjustment of the diode laser array and the entrance slit of the spectrometer. The readout system of the CCD allows integration of the fluorescence light over a preset value, to amplify it and to represent the position of the trapped particles and their fluorescence emission spectra on the vertical and horizontal axes, respectively, of an LCD screen.

The microfluidic system placed at the focal plane of the MO has three inlets and two outlets (Fig. 2). The flow speed in the main channel can be varied between 0 and $100 \mathrm{~mm} / \mathrm{s}$. The whole microfluidic system volume is $\sim 0.5 \mu \mathrm{L}$. The reaction chamber at the center of the system is $500 \mu \mathrm{m}$ wide, $100 \mu \mathrm{m}$ deep, and $500 \mu \mathrm{m}$ long. By changing the pumping rate in outlets $\mathrm{D}$ and $\mathrm{E}$, the flow in the reaction chamber can be steered such that the liquids from inlet $\mathrm{A}, \mathrm{B}$, or $\mathrm{C}$ are directed to either $\mathrm{D}$ or $\mathrm{E}$, which allows particle sorting. In the inset of Fig. 2 , a colored water flow at inlet $B$ is used to illustrate how the liquid stream from $\mathrm{A}$ is directed to $\mathrm{D}$, while the inputs from $\mathrm{B}$ and $\mathrm{C}$ are directed to $\mathrm{E}$. The line of foci from the diode laser light is placed close to the lower wall of the reaction chamber. The angle between the axis of the reaction channel and the line of the focused lasers can be adjusted arbitrarily. It is set at $\sim 45 \mathrm{deg}$ for the following experiments. The focal plane of the diode laser light is situated at a depth of $10-15 \mu \mathrm{m}$.

The transmission of light through the optical system was measured to be $\approx 35 \%$ at $830 \mathrm{~nm}$ (mostly due to the microscope transmission). This allowed us to achieve a maximum power of around $35 \mathrm{~mW}$ per trap and trapping

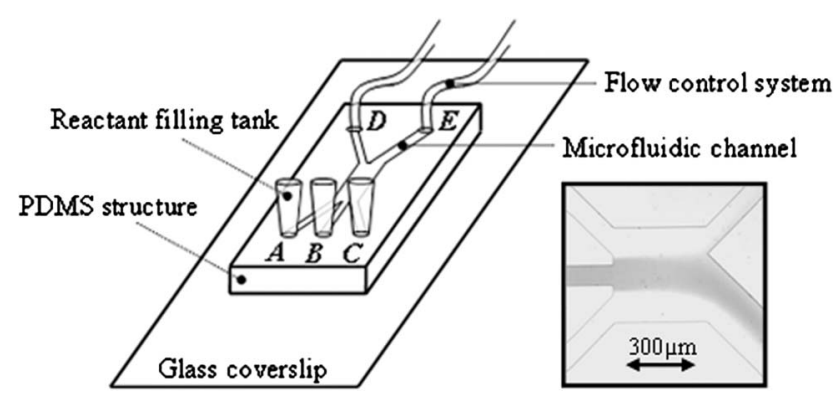

Fig. 2. Microfluidic system. Inset: Control of flow direction illustration. Filling tanks (A, B, C) allow dynamic reactant handling; syringe pumps (D, E) allow accurate flow control. particles in a flow up to $1 \mathrm{~mm} / \mathrm{s}$. Under these conditions, the maximal drag force acting on $6 \mu \mathrm{m}$ diameter polystyrene beads was estimated, according to Stokes' law [1]ㅡ, to be $60 \mathrm{pN}$ per trap.

Figure 3 shows the trapping of beads in the microfluidic channel at a flow rate of $8 \mathrm{nl} / \mathrm{s}(\approx 320 \mu \mathrm{m} / \mathrm{s})$. Four pictures were taken at different time steps $(5 \mathrm{~s}, 10 \mathrm{~s}, 45 \mathrm{~s}$, and $120 \mathrm{~s}$ ) during the process of filling 16 traps. Because of the limited field of view, only one half of the array was used in this experiment. Once trapped, each bead was kept in position during the whole experiment. In order to better observe and record images of this process, we did work neither with maximal flow speed nor at high bead density ( $\approx 50$ beads per $\mu \mathrm{l}$ ). This explains why $2 \mathrm{~min}$ was required to fill all the traps.

The video clip attached to Fig. 4 illustrates both the ability to control traps individually and the possibility of measuring simultaneously the fluorescence of each single trapped bead. Starting with 16 filled traps and a flow rate of $8 \mathrm{nl} / \mathrm{s}$, the video (Media 1) attached to Fig. 4(a) shows the release of chosen particles at a predefined time. Twice during the experiment (at $3 \mathrm{~s}$ and $8 \mathrm{~s}$ ) every other bead is released in the flow of the microfluidic channel, whereas the other half remains trapped. On/off switching times well below $1 \mu \mathrm{s}$ are feasible for individual diodes. This is much faster than the transit time of particles through the trapping zone, i.e., the number of trapping/releasing processes per unit time is limited by the flow condition and the particle density. Figure 4(b) shows spectra of the trapped beads acquired during this experiment at the minimal acquisition time of the spectrometer $(10 \mathrm{~ms})$ with no visible signs of spectral crosstalk. Such effects are also not expected in the case of 64 traps, since the illuminated pixels would still be separated by at least two rows of nonilluminated pixels. The time resolution for monitoring the evolution of the fluorescence spectra of individual trapped particles could be further reduced by using a faster camera.

The maximum intensity of the fluorescence emission varies from bead to bead either due to intrinsic differences between beads or due to inhomogeneous excitation intensity along the trapping line. However, the spectral emission profile could still allow a clear discrimination of different markers (with a spectral resolution $<1 \mathrm{~nm}$ ).
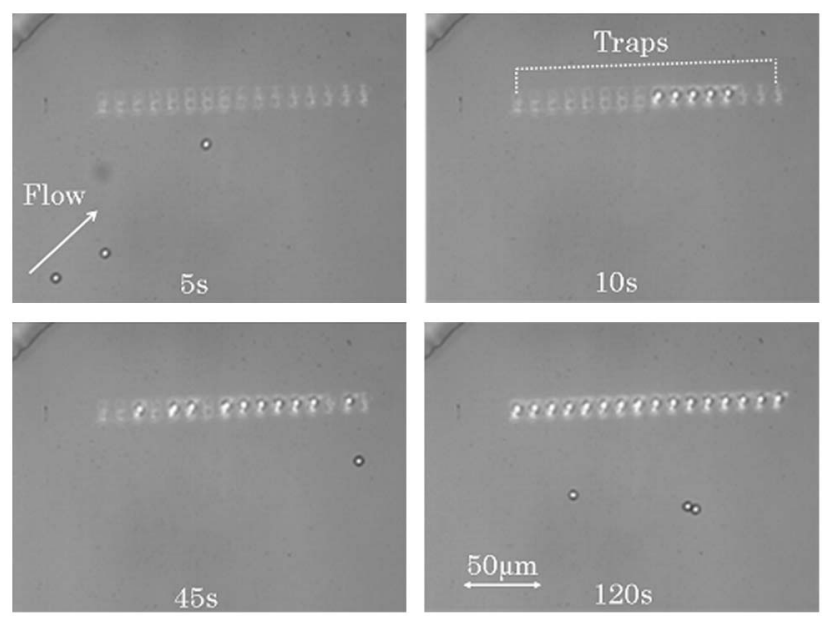

Fig. 3. Steps of the filling traps process. 

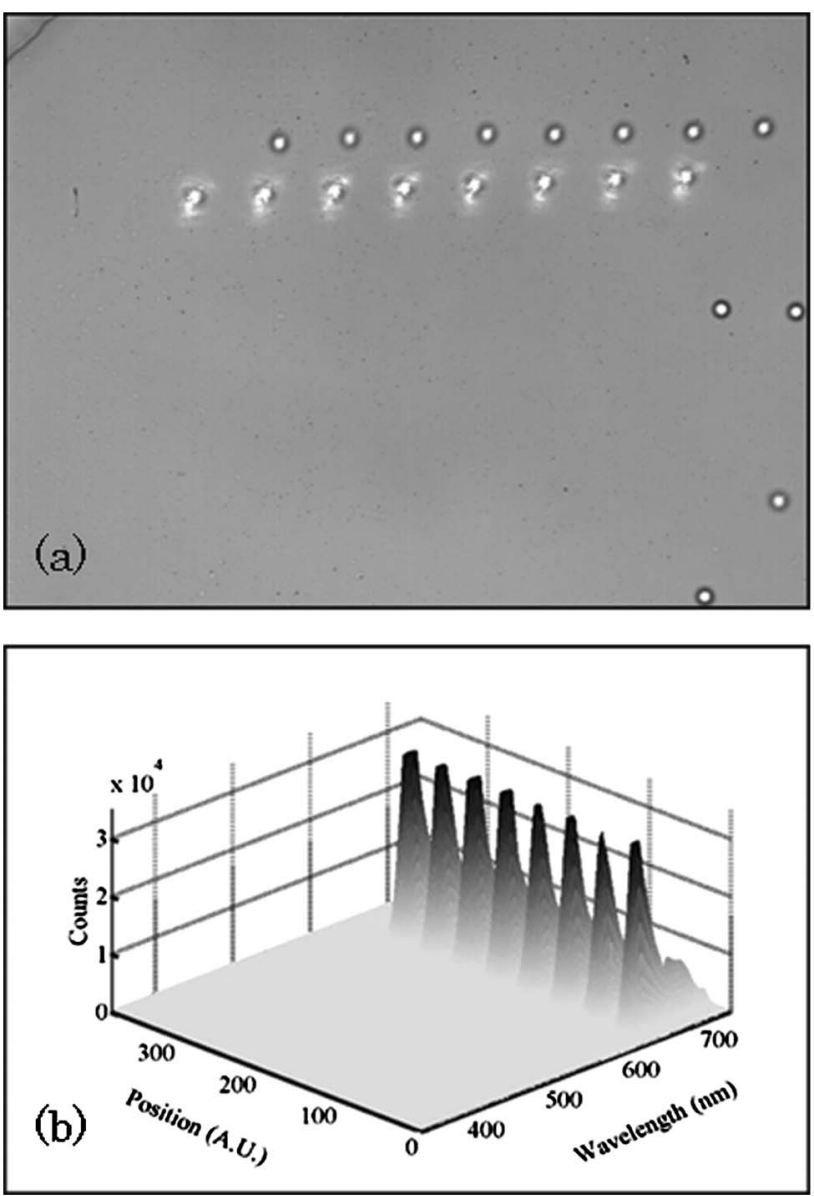

Fig. 4. (a) Individual trap handling and (b) simultaneous fluorescence measurement (Media 1).

The experiment demonstrates the potential of this technique. The combination of individual optical trapping and real time fluorescence spectroscopy over prolonged time periods allows monitoring (bio)chemical reactions of many individual particles. It also shows that fluorescence spectroscopy is actually performed on all the particles trapped in parallel. Depending on their fluorescence spectra, particles can either be kept in the traps for further studies or be released and replaced by other particles. Finally, the spatiotemporal flow control in the microfluidic circuit allows sorting of particles that exhibit interesting properties, e.g., to stock them in different bins.

The consumption of chemicals in such microfluidic circuits is in the microliter range, and the time resolution for monitoring the evolution of fluorescence spectra is essentially limited by the minimum integration time of the CCD camera (10 ms in our case). The rate of handling particles is determined by the maximum number of traps, the flow speed in the reaction channel, and the switching speed of the microfluidic circuit. Upper limits for flow and switching speeds in such experiments are imposed by the maximum force that can be achieved for the trapping. Flow speed of $\sim 1 \mathrm{~mm} / \mathrm{s}$ can be tolerated at a maximum trapping force of $60 \mathrm{pN}$. By taking into account all these parameters, by using the 64 traps, and by optimizing the particle concentration in the solution, one could be able to monitor biochemical reactions of tens of particles per second within a very small volume of solution $(\approx 0.5 \mu \mathrm{L})$. Furthermore, the maximum trapping force could be increased by using more powerful diodes (based on similar modules) and by improving the transmission efficiency of the optical setup.

Finally, the use of directly modulated power diode lasers offers advantages with respect to other multiple trapping techniques. A fast scanning single laser system delivers high power and high trapping forces. Nevertheless, experiments are usually achieved in a static configuration, and even with a very fast transition time within the sites (up to $10 \mu \mathrm{s}$ ), trapping several particles in a flow is severely limited by the laser sweeping time. On the other hand, SLM generated holographic trapping allows trapping of a large number of particles in a perfectly controlled and adjustable 3D pattern, but the duration of dynamical reconfiguration and the achievable forces in the traps limit the speed of moving particles (a few micrometers per second) and make it hard to use in a flow.

In summary, both the interactive capabilities and strong trapping forces of our system offer great advantages with respect to other techniques. The main technology involved remains simple and could easily be improved in terms of power, number of diodes, and automatization. Furthermore, combining parallel trapping with multiple fluorescence spectroscopy offers novel prospects for miniaturized bioanalytics. For instance, the experimental platform can be of interest for the highly parallel (bio)chemical analysis of living cells with the possibility of sorting cells with interesting properties for further investigation. Therefore, potential applications can be found in the areas of clinical diagnostics, drug screening, and toxicity screening.

\section{References}

1. A. Ashkin, Phys. Rev. Lett. 24, 156 (1970).

2. A. Ashkin, J. M. Dziedzic, J. E. Bjorkholm, and S. Chu, Opt. Lett. 11, 288 (1986).

3. D. McGloin, Phil. Trans. R. Soc. A 364, 3521 (2006).

4. E. A. Abbondanzieri, W. J. Greenleaf, J. W. Shaevitz, R. Landick, and S. M. Block, Nature 438, 460 (2005).

5. R. Agarwal, K. Ladavac, Y. Roichman, G. Yu, C. M. Lieber, and D. G. Grier, Opt. Express 13, 8906 (2005).

6. R. W. Applegate Jr., J. Squier, T. Vestad, J. Oakey, and D. W. M. Marr, in Conference on Lasers and Electro-Optics/ Quantum Electronics and Laser Science and Photonic Applications Systems Technologies, OSA Technical Digest (CD) (Optical Society of America, 2005), paper CFN2.

7. E. Fallman and O. Axner, Appl. Opt. 36, 2107 (1997).

8. R. L. Eriksen, P. C. Mogensen, and J. Gluckstad, Opt. Lett. 27, 267 (2002).

9. D. L. J. Vossen, A. van der Horst, M. Dogterom, and A. Van Blaaderen, Rev. Sci. Instrum. 75, 2960 (2004).

10. D. S. Kaputa, A. N. Kuzmin, A. V. Kachynski, A. N. Cartwright, and P. N. Prasad, Appl. Opt. 44, 3963 (2005).

11. P. Zhang, L. Kong, P. Setlow, and Y.-Q. Li, Opt. Lett. 35, 3321 (2010).

12. J. E. Curtis, B. A. Koss, and D. G. Grier, Opt. Commun. 207, 169 (2002).

13. Y. Ogura, K. Kagawa, and J. Tanida, Appl. Opt. 40, 5430 (2001).

14. G. Boer, R. Johann, J. Rohner, F. Merenda, G. Delacrétaz, Ph. Renaud, and R. P. Salathe, Rev. Sci. Instrum. 78, 116101 (2007).

15. W. H. Wright, G. J. Sonek, and M. W. Berns, Appl. Opt. 33, 1735 (1994). 